CRYSTALLOGRAPHIC COMMUNICATIONS

ISSN 2056-9890

Received 30 June 2021

Accepted 2 August 2021

Edited by M. Weil, Vienna University of Technology, Austria

Keywords: crystal structure; 2,3-dihydro-1benzofuran ring system; dimers; hydrogen bonds; Hirshfeld surface analysis.

CCDC reference: 1984938

Supporting information: this article has supporting information at journals.iucr.org/e

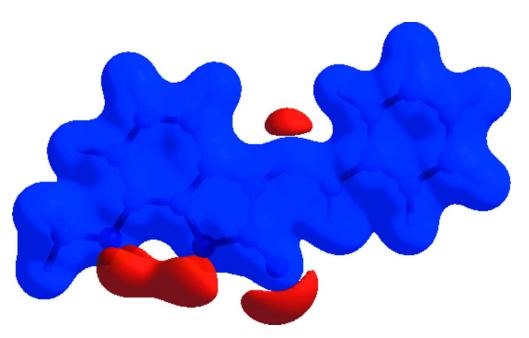

OPEN Ә ACCESS

\section{Crystal structure and Hirshfeld surface analysis of (3Z)-7-methoxy-3-(2-phenylhydrazinylidene)-1- benzofuran-2(3H)-one}

\author{
Zeliha Atioğlu, ${ }^{a}$ Mehmet Akkurt, ${ }^{\text {b }}$ Ulviyya F. Askerova, ${ }^{c}$ Sevinc H. Mukhtarova, ${ }^{c}$ \\ Rizvan K. Askerov ${ }^{\mathrm{c}}$ and Sixberth Mlowe ${ }^{\mathrm{d} *}$
}

\begin{abstract}
aDepartment of Aircraft Electrics and Electronics, School of Applied Sciences, Cappadocia University, Mustafapaşa, 50420 Ürgüp, Nevşehir, Turkey, 'b Department of Physics, Faculty of Sciences, Erciyes University, 38039 Kayseri, Turkey, ' Organic Chemistry Department, Baku State University, Z. Xalilov str. 23, Az, 1148 Baku, Azerbaijan, and dUniversity of Dar es Salaam, Dar es Salaam University College of Education, Department of Chemistry, PO Box 2329, Dar es Salaam, Tanzania. *Correspondence e-mail: sixberth.mlowe@duce.ac.tz
\end{abstract}

In the title compound, $\mathrm{C}_{15} \mathrm{H}_{12} \mathrm{~N}_{2} \mathrm{O}_{3}$, pairs of molecules are linked into dimers by $\mathrm{N}-\mathrm{H} \cdots \mathrm{O}$ hydrogen bonds, forming an $R_{2}^{2}(12)$ ring motif, with the dimers stacked along the $a$ axis. These dimers are connected through $\pi-\pi$ stacking interactions between the centroids of the benzene and furan rings of their 2,3-dihydro-1-benzofuran ring systems. Furthermore, there exists a $\mathrm{C}-\mathrm{H} \cdots \pi$ interaction that consolidates the crystal packing. A Hirshfeld surface analysis indicates that the most important contacts are $\mathrm{H} \cdots \mathrm{H}(40.7 \%), \mathrm{O} \cdots \mathrm{H} / \mathrm{H} \cdots \mathrm{O}$ $(24.7 \%), \mathrm{C} \cdots \mathrm{H} / \mathrm{H} \cdots \mathrm{C}(16.1 \%)$ and $\mathrm{C} \cdots \mathrm{C}(8.8 \%)$.

\section{Chemical context}

Hydrazones are a versatile class of organic ligands that have extensive applications in synthetic transformations, the synthesis of bioactive compounds, the design of materials and in coordination chemistry (Ma et al., 2017a,b; Viswanathan et al., 2019). Moreover, metal complexes of hydrazone ligands have been successfully applied as catalysts in organic synthesis (Gurbanov et al., 2018). The properties of metal-hydrazonates can be regulated by the design of ligands through the involvement of non-covalent-bond donor or acceptor substituents (Ma et al., 2020, 2021; Mahmudov et al., 2013). Supramolecular networks of all dimensions in the crystal structures of hydrazone compounds or metal-hydrazonates, resulting from extensive hydrogen-bonding and other types of intermolecular interactions, have been reported (Gurbanov et al., 2020a; Kopylovich et al., 2011). Thus, the attachment of suitable substituents or synthons to hydrazone ligands can improve their functional properties and the catalytic or biological activity of the corresponding coordination compounds (Mizar et al., 2012; Gurbanov et al., 2020a,b; Khalilov et al., 2018a,b; Maharramov et al., 2018; Shihkaliyev et al., 2019; Shixaliyev et al., 2014).<smiles>COc1cccc2c1OC(=O)/C2=N\Nc1ccccc1</smiles> 


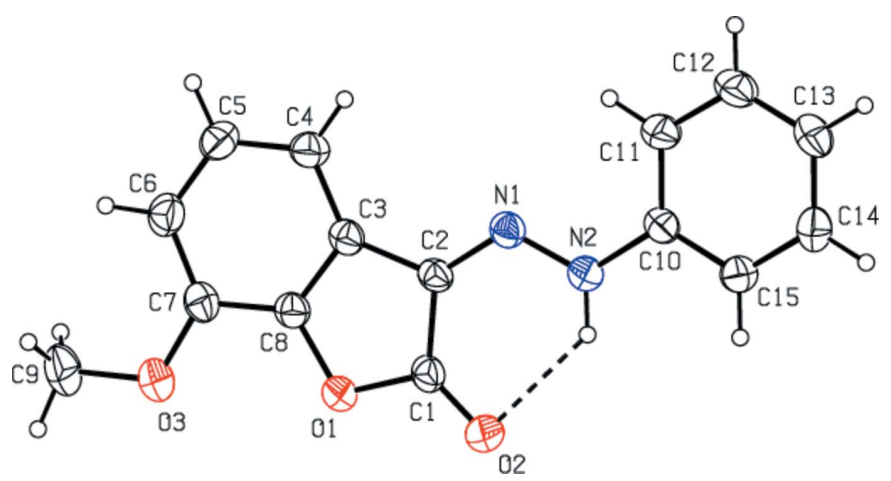

Figure 1

The title molecule with the labelling scheme and displacement ellipsoids drawn at the $30 \%$ probability level. The intramolecular $\mathrm{N}-\mathrm{H} \cdots \mathrm{O}$ hydrogen bond is shown as a dashed line.

In a continuation of our work in this context (Atioğlu et al., $2020,2021)$, we have synthesized a new hydrazone compound, (3Z)-7-methoxy-3-(2-phenylhydrazinylidene)-1-benzofuran$2(3 H)$-one, which shows multiple intermolecular non-covalent interactions.

\section{Structural commentary}

In the title compound, the molecular conformation is stabilized by an intramolecular $\mathrm{N} 2-\mathrm{H} 1 \cdots \mathrm{O} 2$ hydrogen bond, forming an $S(6)$ ring motif (Table 1, Fig. 1; Bernstein et al., 1995). The 2,3-dihydro-1-benzofuran ring system (O1/C1-C8) is essentially planar [maximum deviation of 0.016 (2) $\AA$ for O1] and subtends a dihedral angle of $5.32(14)^{\circ}$ with the phenyl ring (C10-C15).

\section{Supramolecular features}

In the crystal, pairs of molecules are linked into dimers by intermolecular $\mathrm{N}-\mathrm{H}$. . O hydrogen bonds, forming an $R_{2}^{2}(12)$

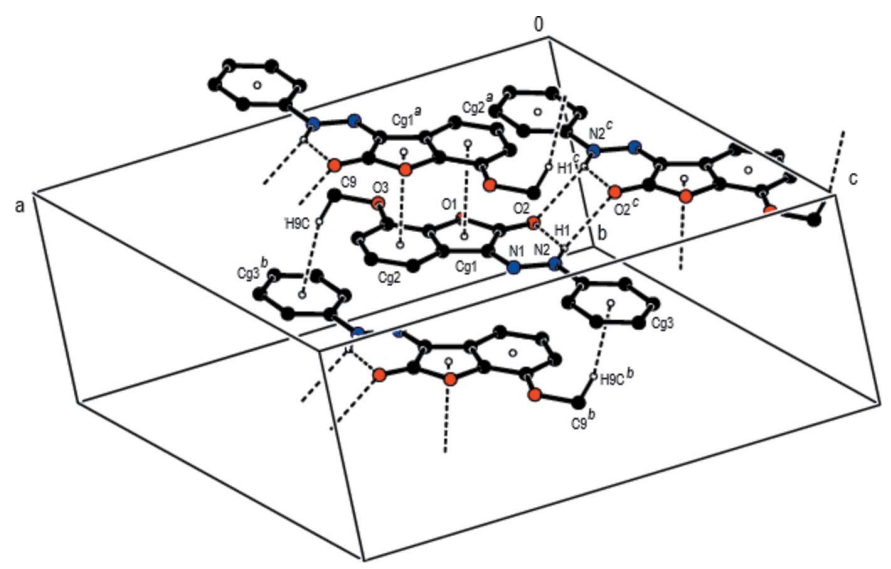

Figure 2

Intermolecular $\mathrm{N}-\mathrm{H} \cdots \mathrm{O}$ hydrogen bonds, $\mathrm{C}-\mathrm{H} \cdots \pi$ interactions and $\pi-\pi$ stacking interactions (shown as dashed lines) in the title compound. [Symmetry codes: (a) $1-x,-y, 1-z ;(b) 1-x, 1-y, 1-z ;(c) \frac{1}{2}-x$, $\left.\frac{1}{2}-y, 1-z\right]$.
Table 1

Hydrogen-bond geometry $\left(\AA,^{\circ}\right)$.

$\mathrm{Cg} 3$ is the centroid of the $\mathrm{C} 10-\mathrm{C} 15$ phenyl ring.

\begin{tabular}{lllll}
\hline$D-\mathrm{H} \cdots A$ & $D-\mathrm{H}$ & $\mathrm{H} \cdots A$ & $D \cdots A$ & $D-\mathrm{H} \cdots A$ \\
\hline $\mathrm{N} 2-\mathrm{H} 1 \cdots \mathrm{O} 2$ & $0.92(4)$ & $2.14(3)$ & $2.843(3)$ & $133(3)$ \\
$\mathrm{N} 2-\mathrm{H} 1 \cdots \mathrm{O} 2^{\mathrm{i}}$ & $0.92(4)$ & $2.44(4)$ & $3.181(4)$ & $138(3)$ \\
$\mathrm{C} 9-\mathrm{H} 9 C \cdots C g 3^{\mathrm{ii}}$ & 0.96 & 2.70 & $3.555(4)$ & 149 \\
\hline
\end{tabular}

Symmetry codes: (i) $-x+\frac{1}{2},-y+\frac{1}{2},-z+1$; (ii) $1-x, 1-y, 1-z$.

ring motif (Table 1). These dimers are stacked along the $a$ axis and connected by $\pi-\pi$ stacking interactions between the centroids of the benzene and furan rings of their 2,3-dihydro1-benzofuran ring systems $[C g 1 \cdots C g 2(1-x,-y, 1-z)=$ 3.5316 (19) $\AA$, slippage $=0.352 \AA$, where $C g 1$ and $C g 2$ are the centroids of the benzene $(\mathrm{C} 3-\mathrm{C} 8)$ and furan $(\mathrm{O} 1 / \mathrm{C} 1-\mathrm{C} 3 / \mathrm{C} 8)$ rings, respectively] (Figs. 2, 3 and 4). Furthermore, there exists a $\mathrm{C}-\mathrm{H} \cdots \pi$ interaction between the $\mathrm{H} 9 \mathrm{C}$ atom of the methyl group C9 and the centroid of the phenyl ring ( $\mathrm{C} 10-\mathrm{C} 15)$.

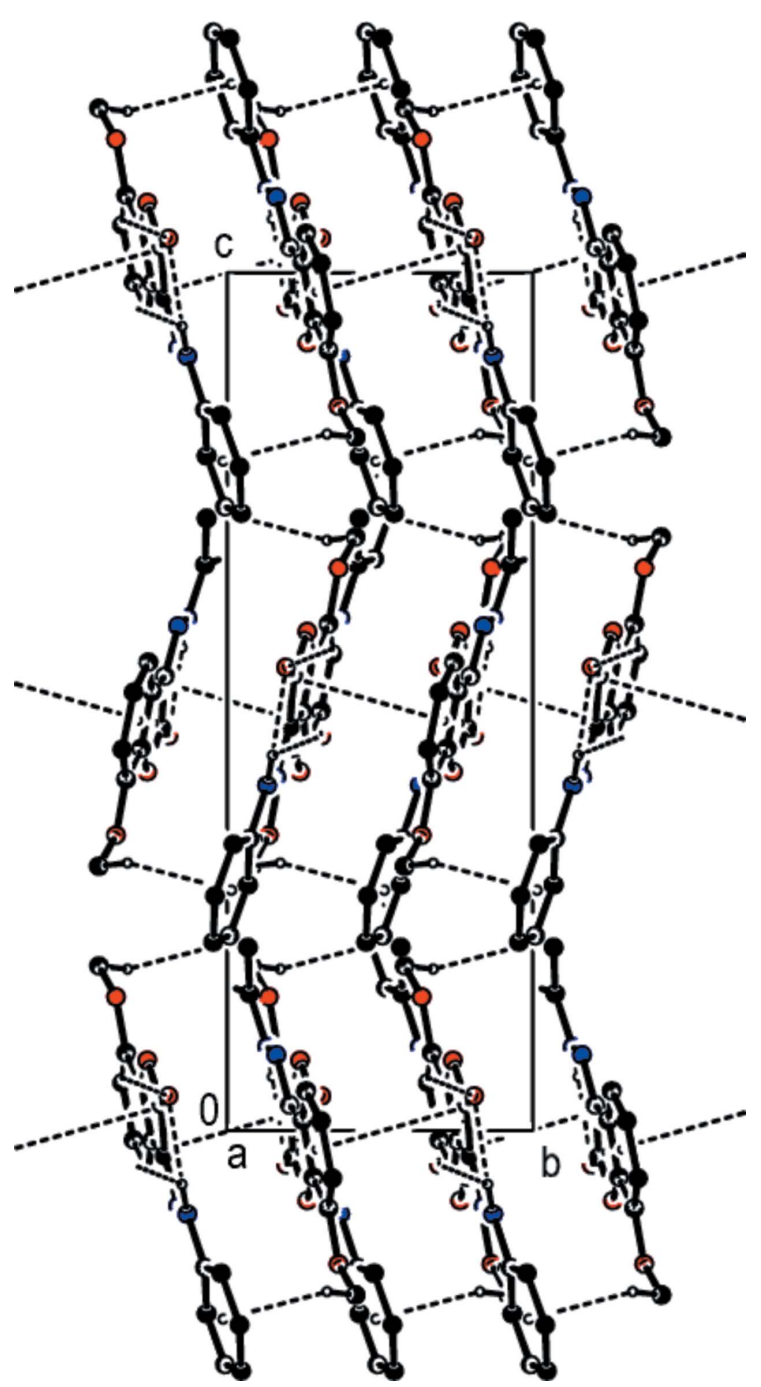

Figure 3

A view of the molecular packing of the title compound along the $a$-axis direction. Intermolecular interactions are depicted as in Fig. 2. 


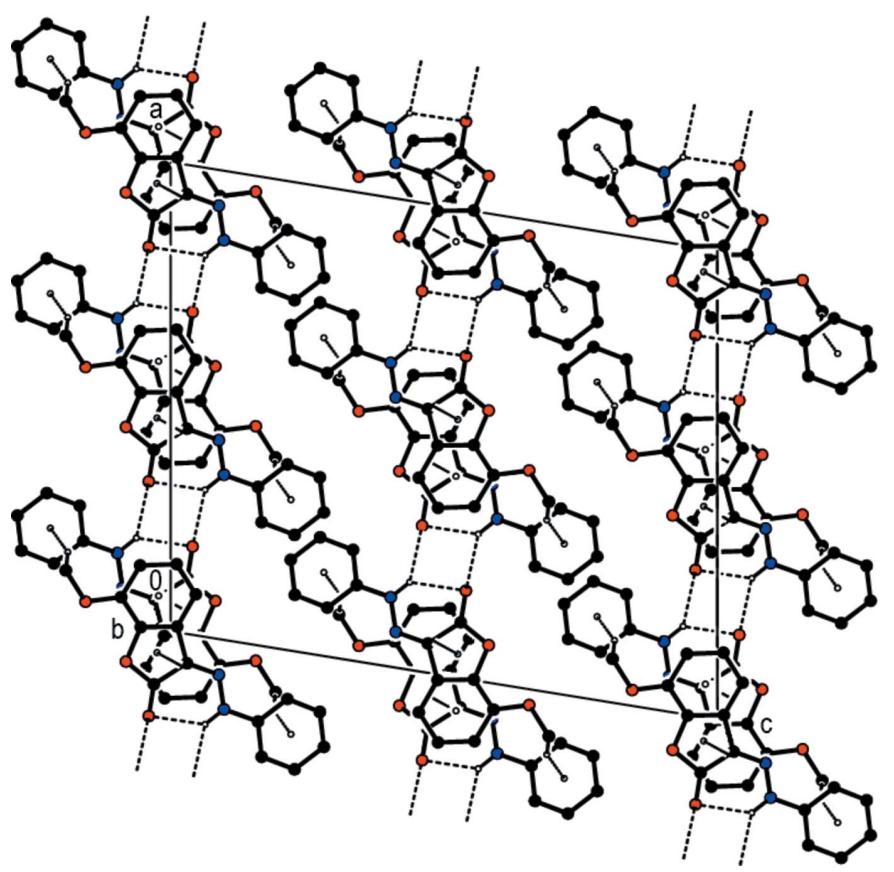

Figure 4

A view of the molecular packing of the title compound along the $b$-axis direction. Intermolecular interactions are depicted as in Fig. 2.

\section{Hirshfeld surface analysis}

Crystal Explorer 17.5 (Turner et al., 2017) was used to calculate the Hirshfeld surfaces and generate the two-dimensional fingerprint plots. Hirshfeld surfaces allow for the display of intermolecular interactions by using distinct colours and intensities to indicate short and long contacts, as well as the

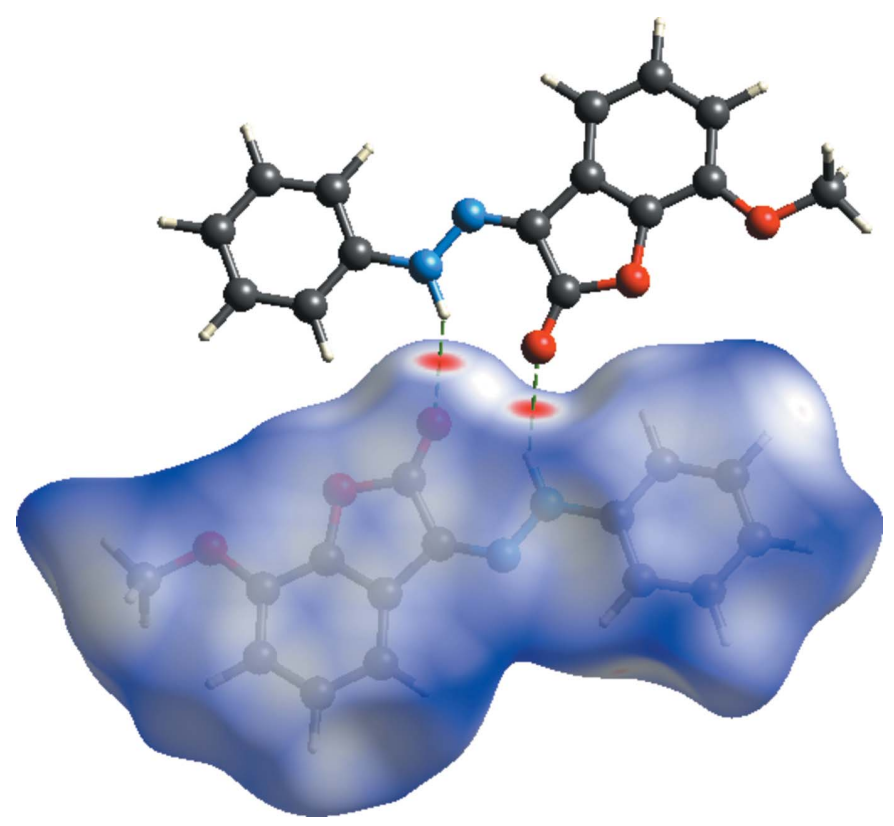

Figure 5

View of the three-dimensional Hirshfeld surface of the title compound plotted over $d_{\text {norm }}$ in the range -0.1718 to 1.3843 a.u. The two $\mathrm{N}-\mathrm{H} \cdots \mathrm{O}$ hydrogen bonds forming the dimer are depicted as dashed lines.
Table 2

Interatomic contacts of the title compound $(\AA)$.

\begin{tabular}{lll}
\hline Contact & Distance & Symmetry operation \\
\hline $\mathrm{H} 1 \cdots \mathrm{O} 2$ & 2.44 & $\frac{1}{2}-x, \frac{1}{2}-y, 1-z$ \\
$\mathrm{H} 9 B \cdots \mathrm{N} 2$ & 2.91 & $1-x,-y, 1-z$ \\
$\mathrm{H} 9 C \cdots \mathrm{C} 11$ & 2.93 & $1-x, 1-y, 1-z$ \\
$\mathrm{H} 5 A \cdots \mathrm{H} 15 A$ & 2.51 & $\frac{1}{2}+x,-\frac{1}{2}+y, z$ \\
$\mathrm{C} 9 \cdots \mathrm{H} 14 A$ & 2.85 & $\frac{1}{2}+x, \frac{1}{2}-y,-\frac{1}{2}+z$ \\
$\mathrm{H} 11 A \cdots \mathrm{H} 11 A$ & 2.31 & $1-x, y, \frac{3}{2}-z$ \\
$\mathrm{C} 15 \cdots \mathrm{H} 13 A$ & 3.07 & $\frac{1}{2}-x,-\frac{1}{2}+y, \frac{3}{2}-z$ \\
\hline
\end{tabular}

relative strength of the interactions. The three-dimensional Hirshfeld surface of the title compound plotted over $d_{\text {norm }}$ in the range -0.1718 to 1.3843 a.u. is shown in Fig. 5. The N2$\mathrm{H} 1 \cdots \mathrm{O} 2$ interactions, which play a key role in the molecular packing of the title compound, are responsible for the red spot that occurs around $\mathrm{O} 2$. The bright-red spots appearing near $\mathrm{O} 2$ and hydrogen atom $\mathrm{H} 1$ indicate their roles as donors and/ or acceptors in hydrogen-bonding; they also appear as blue and red regions corresponding to positive and negative potentials on the Hirshfeld surface mapped over electrostatic potential (Spackman et al., 2008) shown in Fig. 6. Here the blue regions indicate positive electrostatic potential (hydrogen-bond donors), while the red regions indicate negative electrostatic potential (hydrogen-bond acceptors).

The overall two-dimensional fingerprint plot for the title compound is given in Fig. $7 a$, and those delineated into $\mathrm{H} \cdots \mathrm{H}$, $\mathrm{O} \cdots \mathrm{H} / \mathrm{H} \cdots \mathrm{O}, \mathrm{C} \cdots \mathrm{H} / \mathrm{H} \cdots \mathrm{C}$ and $\mathrm{C} \cdots \mathrm{C}$ contacts are shown in Fig. $7 b-e$, while numerical details of the different contacts are given in Table 2. The percentage contributions to the Hirshfeld surfaces from the various interatomic contacts are as follows: $\mathrm{H} \cdots \mathrm{H}$ (Fig. $7 b ; 40.7 \%$ ), $\mathrm{O} \cdots \mathrm{H} / \mathrm{H} \cdots \mathrm{O}$ (Fig. 7c; $24.7 \%$ ), C. $\cdots \mathrm{H} / \mathrm{H} \cdots \mathrm{C}$ (Fig. 7d; 16.1\%) and C..C (Fig. 7e; $8.8 \%)$. Other minor contributions to the Hirshfeld surface are from $\mathrm{N} \cdots \mathrm{C} / \mathrm{C} \cdots \mathrm{N}(3.8 \%), \mathrm{N} \cdots \mathrm{H} / \mathrm{H} \cdots \mathrm{N} \quad(3.5 \%), \mathrm{O} \cdots \mathrm{C} /$ $\mathrm{C} \cdots \mathrm{O}(1.9 \%), \mathrm{O} \cdots \mathrm{N} / \mathrm{N} \cdots \mathrm{O}(0.4 \%)$ and $\mathrm{O} \cdots \mathrm{O}(0.2 \%)$ contacts.

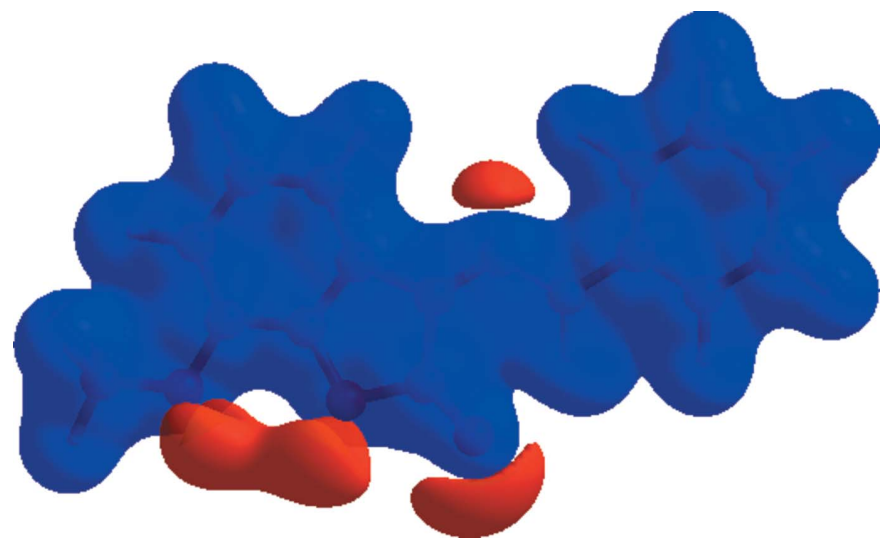

Figure 6

View of the three-dimensional Hirshfeld surface of the title compound plotted over electrostatic potential energy in the range -0.0500 to 0.0500 a.u. using the STO-3 G basis set at the Hartree-Fock level of theory. The hydrogen-bond donors and acceptors are viewed as blue and red regions, respectively, around atoms, corresponding to positive and negative potentials. 


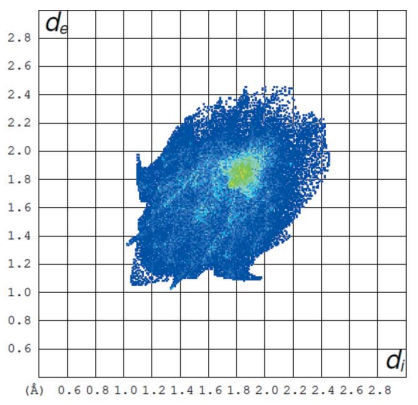

(a) All...All

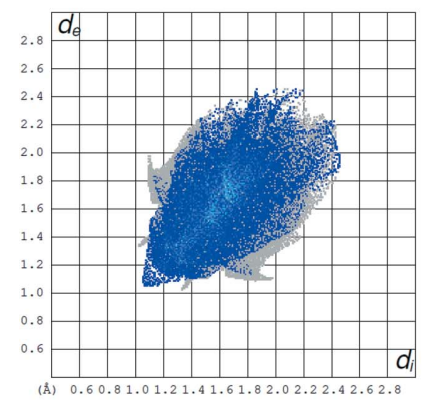

(b) $H \ldots H$

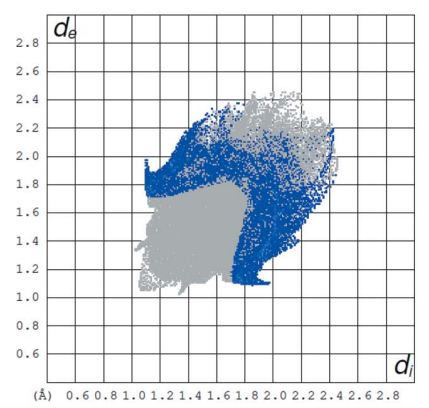

(d) $\mathrm{C} \cdots H / H \cdots C$

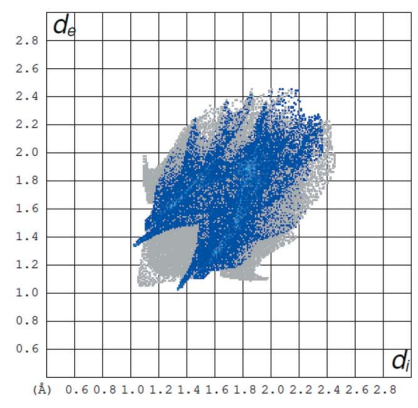

(c) $\mathrm{O} \cdots \mathrm{H} / \mathrm{H} \cdots \mathrm{O}$

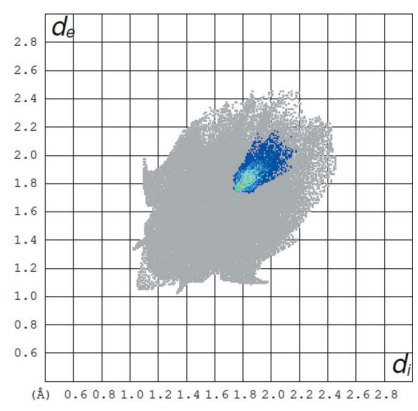

(e) $C \cdots C$
Figure 7

The full two-dimensional fingerprint plots for the title compound, showing $(a)$ all interactions, and delineated into $(b) \mathrm{H} \cdots \mathrm{H},(c) \mathrm{O} \cdots \mathrm{H} /$ $\mathrm{H} \cdots \mathrm{O},(d) \mathrm{C} \cdots \mathrm{H} / \mathrm{H} \cdots \mathrm{C}$ and $(e) \mathrm{C} \cdots \mathrm{C}$ interactions. The $d_{\mathrm{i}}$ and $d_{\mathrm{e}}$ values are the closest internal and external distances (in $\AA$ ) from given points on the Hirshfeld surface contacts.

\section{Database survey}

A search of the Cambridge Crystallographic Database (CSD version 5.40, update of September 2019; Groom et al., 2016) gave 763 hits for structures with a hydrazone moiety. Five structures that are closely related to the title compound are: 2-(4-nitro-1 $H$-imidazol-1-yl)- $N^{\prime}$-[1-(pyridin-2-yl)ethylidene]acetohydrazide (TODMEH; Oliveira et al., 2019); 2-(2-nitro$1 H$-imidazol-1-yl)- $N^{\prime}$-[1-(pyridin-2-yl)ethylidene]acetohydrazide (TODMIL; Oliveira et al., 2019); 2-(4-nitro- $1 H$-imidazol1-yl)- $N^{\prime}$-[phenyl(pyridin-2-yl)methylidene]acetohydrazide (TODMOR; Oliveira et al., 2019); 2-(4-nitro-1H-imidazol-1yl)- $N^{\prime}$-[phenyl(pyridin-2-yl)methylidene]acetohydrazide (TODMUX; Oliveira et al., 2019) and 1,1'-[1,3-phenyl- enebis(2,2-dichloroethene-1,1-diyl)]bis(phenyldiazene) (EXIWOA; Shikhaliyev et al., 2021).

TODMEH and TODMOR crystallize in the monoclinic space group $P 2_{1} / c$ with $Z=4$. TODMIL crystallizes in the monoclinic space group $I 2 / a$ with $Z=8$ and TODMUX crystallizes in the triclinic space group $P \overline{1}$ with $Z=2$. EXIWOA crystallizes in the monoclinic space group $P 2_{1} / \mathrm{c}$ with $Z=4$. The $E$ conformation in TODMEH, TODMIL and TODMUX is stabilized by a strong intermolecular $\mathrm{N}-\mathrm{H} \cdots \mathrm{O}$ interaction. These interactions lead to the formation of dimeric structural arrangements. In the crystal packing of TODMOR, an intermolecular $\mathrm{N}-\mathrm{H} \cdots \mathrm{N}$ interaction results in a zigzag structural arrangement, with the formation of chains along the crystallographic $b$ axis. Non-classical intermolecular $\mathrm{C}-\mathrm{H} \cdots \mathrm{N}$ and $\mathrm{C}-\mathrm{H} \cdots \mathrm{O}$ interactions are also observed in the crystal structures of TODMEH, TODMIL, TODMOR and TODMUX. In EXIWOA, molecules are linked by $\mathrm{C}-\mathrm{H} \cdots \pi, \mathrm{C}-\mathrm{Cl} \cdots \pi$, $\mathrm{Cl} \cdots \mathrm{Cl}$ and $\mathrm{Cl} \cdots \mathrm{H}$ interactions, forming a three-dimensional supramolecular network.

\section{Synthesis and crystallization}

A $20 \mathrm{ml}$ screw-neck vial was charged with dimethyl sulfoxide

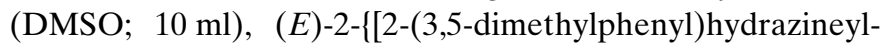
idene]methyl $\}$ phenol $(240 \mathrm{mg}, 1 \mathrm{mmol})$, tetramethylethylenediamine (TMEDA; $295 \mathrm{mg}, 2.5 \mathrm{mmol}), \mathrm{CuCl}(2 \mathrm{mg}$, $0.02 \mathrm{mmol}$ ) and $\mathrm{CCl}_{4}(20 \mathrm{mmol}, 10$ equiv). After $1-3 \mathrm{~h}$ (until TLC analysis showed complete consumption of the corresponding Schiff base), the reaction mixture was poured into a $0.01 M$ solution of $\mathrm{HCl}(100 \mathrm{~mL}, \mathrm{pH}=2-3)$, and extracted with dichloromethane $(3 \times 20 \mathrm{ml})$. The combined organic phase was washed with water $(3 \times 50 \mathrm{ml})$, brine $(30 \mathrm{ml})$, dried over anhydrous $\mathrm{Na}_{2} \mathrm{SO}_{4}$ and concentrated in vacuo in a rotary evaporator. The residue was purified by column chromatography on silica gel using appropriate mixtures of hexane and dichloromethane $(v / v=3 / 1-1 / 1)$. Colourless solid (yield $65 \%)$; m.p. $475 \mathrm{~K}$. Analysis calculated for $\mathrm{C}_{15} \mathrm{H}_{12} \mathrm{~N}_{2} \mathrm{O}_{3}(M=268.27)$ : C 67.16, H 4.51, N 10.44; found: C 67.11, H 4.47, N 10.35\%. ${ }^{1} \mathrm{H}$ NMR $\left(300 \mathrm{MHz}, \mathrm{CDCl}_{3}\right) \delta 12.13(s, 1 \mathrm{H}, \mathrm{NH}), 6.91-7.43(8 \mathrm{H}$, Ar), $3.99\left(s, 3 \mathrm{H}, \mathrm{OCH}_{3}\right) .{ }^{13} \mathrm{C} \mathrm{NMR}\left(75 \mathrm{MHz} \mathrm{CDCl}_{3}\right) \delta 186.20$, 161.87, 150.65, 141.76, 129.60, 125.09, 124.44, 123.87, 114.90, 112.74, 111.44, 108.76, 56.46. ESI-MS: $m / z: 269.26[M+\mathrm{H}]^{+}$. Crystals suitable for X-ray analysis were obtained by slow evaporation of a dichloromethane solution.

\section{Refinement details}

Crystal data, data collection and structure refinement details are summarized in Table 3. The $\mathrm{H}$ atom of the $\mathrm{NH}$ group was located in a difference-Fourier map and refined freely [N2$\mathrm{H} 1=0.92$ (4) $\AA$ ]. $\mathrm{H}$ atoms bonded to $\mathrm{C}$ atoms were positioned geometrically and refined using a riding model, with $\mathrm{C}-\mathrm{H}=$ 0.93 or $0.96 \AA$, and with $U_{\text {iso }}(\mathrm{H})=1.2 U_{\text {eq }}(\mathrm{C})$ for aromatic or $1.5 U_{\text {eq }}(\mathrm{C})$ for methyl $\mathrm{H}$ atoms. Owing to poor agreement between observed and calculated intensities, seven outliers,

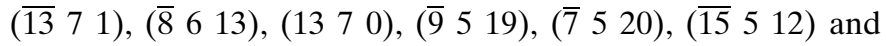
(0 6 16), were omitted in the final cycles of refinement. 
Table 3

Experimental details.

\begin{tabular}{ll}
\hline Crystal data & \\
Chemical formula & $\mathrm{C}_{15} \mathrm{H}_{12} \mathrm{~N}_{2} \mathrm{O}_{3}$ \\
$M_{\mathrm{r}}$ & 268.27 \\
Crystal system, space group & Monoclinic, $C 2 / c$ \\
Temperature (K) & 296 \\
$a, b, c(\AA)$ & $17.436(2), 7.2485(7), 20.595(2)$ \\
$\beta\left({ }^{\circ}\right.$ & $99.181(4)$ \\
$V\left(\AA^{3}\right)$ & $2569.6(5)$ \\
$Z$ & 8 \\
Radiation type & Mo $K \alpha$ \\
$\mu\left(\mathrm{mm}^{-1}\right)$ & 0.10 \\
Crystal size (mm) & $0.49 \times 0.15 \times 0.06$ \\
& \\
Data collection & Bruker APEXII CCD \\
Diffractometer & Multi-scan $(S A D A B S ;$ Krause $e t$ \\
Absorption correction & al., 2015) \\
& $0.629,0.745$ \\
$T_{\text {min }}, T_{\text {max }}$ & $12800,2427,1463$ \\
No. of measured, independent and & \\
$\quad$ observed $[I>2 \sigma(I)]$ reflections & 0.085 \\
$R_{\text {int }}$ & 0.616 \\
$(\text { sin } \theta / \lambda)_{\text {max }}\left(\AA^{-1}\right)$ & \\
Refinement & \\
$R\left[F^{2}>2 \sigma\left(F^{2}\right)\right], w R\left(F^{2}\right), S$ & $0.073,0.152,1.01$ \\
No. of reflections & 2427 \\
No. of parameters & 187 \\
$\mathrm{H}$-atom treatment & $\mathrm{H}$ atoms treated by a mixture of \\
& independent and constrained \\
$\Delta \rho_{\text {max }}, \Delta \rho_{\text {min }}\left(\mathrm{e} \AA^{-3}\right)$ & refinement \\
\hline
\end{tabular}

Computer programs: APEX3 and SAINT (Bruker, 2017), SHELXS (Sheldrick, 2008), SHELXL (Sheldrick, 2015), ORTEP-3 for Windows (Farrugia, 2012) and PLATON (Spek, 2020).

\section{Acknowledgements}

The authors' contributions are as follows. Conceptualization, MA and UFA; methodology, ZA and SHM; investigation, SHM, RKA, and ZA; writing (original draft), MA and SM; writing (review and editing of the manuscript), MA and UFA; visualization, RKA, ZA and MA; funding acquisition, UFA, SHM and RKA; resources, RKA, ZA and SHM; supervision, MA and SM.

\section{Funding information}

This work was performed under the support of the Science Development Foundation under the President of the Republic of Azerbaijan (grant No. EIF-BGM-4- RFTF-1/2017-21/13/4).

\section{References}

Atioğlu, Z., Akkurt, M., Shikhaliyev, N. Q., Askerova, U. F., Niyazova, A. A. \& Mlowe, S. (2021). Acta Cryst. E77, 829-833.

Atioğlu, Z., Akkurt, M., Shikhaliyev, N. Q., Suleymanova, G. T., Babayeva, G. V., Gurbanova, N. V., Mammadova, G. Z. \& Mlowe, S. (2020). Acta Cryst. E76, 1291-1295.

Bernstein, J., Davis, R. E., Shimoni, L. \& Chang, N.-L. (1995). Angew. Chem. Int. Ed. Engl. 34, 1555-1573.

Bruker (2017). APEX2 and SAINT. Bruker AXS Inc., Madison, Wisconsin, USA.
Farrugia, L. J. (2012). J. Appl. Cryst. 45, 849-854.

Groom, C. R., Bruno, I. J., Lightfoot, M. P. \& Ward, S. C. (2016). Acta Cryst. B72, 171-179.

Gurbanov, A. V., Kuznetsov, M. L., Demukhamedova, S. D., Alieva, I. N., Godjaev, N. M., Zubkov, F. I., Mahmudov, K. T. \& Pombeiro, A. J. L. (2020a). CrystEngComm, 22, 628-633.

Gurbanov, A. V., Kuznetsov, M. L., Mahmudov, K. T., Pombeiro, A. J. L. \& Resnati, G. (2020b). Chem. Eur. J. 26, 14833-14837.

Gurbanov, A. V., Maharramov, A. M., Zubkov, F. I., Saifutdinov, A. M. \& Guseinov, F. I. (2018). Aust. J. Chem. 71, 190-194.

Khalilov, A. N., Asgarova, A. R., Gurbanov, A. V., Maharramov, A. M., Nagiyev, F. N. \& Brito, I. (2018a). Z. Kristallogr. New Cryst. Struct. 233, 1019-1020.

Khalilov, A. N., Asgarova, A. R., Gurbanov, A. V., Nagiyev, F. N. \& Brito, I. (2018b). Z. Kristallogr. New Cryst. Struct. 233, 947-948.

Kopylovich, M. N., Mahmudov, K. T., Mizar, A. \& Pombeiro, A. J. L. (2011). Chem. Commun. 47, 7248-7250.

Krause, L., Herbst-Irmer, R., Sheldrick, G. M. \& Stalke, D. (2015). J. Appl. Cryst. 48, 3-10.

Ma, Z., Gurbanov, A. V., Maharramov, A. M., Guseinov, F. I., Kopylovich, M. N., Zubkov, F. I., Mahmudov, K. T. \& Pombeiro, A. J. L. (2017a). J. Mol. Catal. A Chem. 426, 526-533.

Ma, Z., Gurbanov, A. V., Sutradhar, M., Kopylovich, M. N., Mahmudov, K. T., Maharramov, A. M., Guseinov, F. I., Zubkov, F. I. \& Pombeiro, A. J. L. (2017b). Mol. Catal. 428, 17-23.

Ma, Z., Mahmudov, K. T., Aliyeva, V. A., Gurbanov, A. V., Guedes da Silva, M. F. C. \& Pombeiro, A. J. L. (2021). Coord. Chem. Rev. 437, 213859.

Ma, Z., Mahmudov, K. T., Aliyeva, V. A., Gurbanov, A. V. \& Pombeiro, A. J. L. (2020). Coord. Chem. Rev. 423, 213482.

Maharramov, A. M., Shikhaliyev, N. Q., Suleymanova, G. T., Gurbanov, A. V., Babayeva, G. V., Mammadova, G. Z., Zubkov, F. I., Nenajdenko, V. G., Mahmudov, K. T. \& Pombeiro, A. J. L. (2018). Dyes Pigments, 159, 135-141.

Mahmudov, K. T., Kopylovich, M. N., Haukka, M., Mahmudova, G. S., Esmaeila, E. F., Chyragov, F. M. \& Pombeiro, A. J. L. (2013). J. Mol. Struct. 1048, 108-112.

Mizar, A., Guedes da Silva, M. F. C., Kopylovich, M. N., Mukherjee, S., Mahmudov, K. T. \& Pombeiro, A. J. L. (2012). Eur. J. Inorg. Chem. pp. 2305-2313.

Oliveira, A. P. A., Ferreira, I. P., Despaigne, A. A. R., Silva, J. G. da, Vieira, A. C. S., Santos, M. S., Alexandre-Moreira, M. S., Diniz, R. \& Beraldo, H. (2019). Acta Cryst. C75, 320-328.

Sheldrick, G. M. (2008). Acta Cryst. A64, 112-122.

Sheldrick, G. M. (2015). Acta Cryst. C71, 3-8.

Shikhaliyev, N. Q., Atioğlu, Z., Akkurt, M., Ahmadova, N. E., Askerov, R. K. \& Bhattarai, A. (2021). Acta Cryst. E77, 814-818.

Shikhaliyev, N. Q., Kuznetsov, M. L., Maharramov, A. M., Gurbanov, A. V., Ahmadova, N. E., Nenajdenko, V. G., Mahmudov, K. T. \& Pombeiro, A. J. L. (2019). CrystEngComm, 21, 5032-5038.

Shixaliyev, N. Q., Gurbanov, A. V., Maharramov, A. M., Mahmudov, K. T., Kopylovich, M. N., Martins, L. M. D. R. S., Muzalevskiy, V. M., Nenajdenko, V. G. \& Pombeiro, A. J. L. (2014). New J. Chem. 38, 4807-4815.

Spackman, M. A., McKinnon, J. J. \& Jayatilaka, D. (2008). CrystEngComm, 10, 377-388.

Spek, A. L. (2020). Acta Cryst. E76, 1-11.

Turner, M. J., McKinnon, J. J., Wolff, S. K., Grimwood, D. J., Spackman, P. R., Jayatilaka, D. \& Spackman, M. A. (2017). CrystalExplorer17. The University of Western Australia.

Viswanathan, A., Kute, D., Musa, A., Mani, S. K., Sipilä, V., EmmertStreib, F., Zubkov, F. I., Gurbanov, A. V., Yli-Harja, O. \& Kandhavelu, M. (2019). Eur. J. Med. Chem. 166, 291-303. 


\section{supporting information}

Acta Cryst. (2021). E77, 907-911［https://doi.org/10.1107/S2056989021007891]

Crystal structure and Hirshfeld surface analysis of (3Z)-7-methoxy-3-(2-phenylhydrazinylidene)-1-benzofuran-2(3H)-one

\section{Zeliha Atioğlu, Mehmet Akkurt, Ulviyya F. Askerova, Sevinc H. Mukhtarova, Rizvan K.}

Askerov and Sixberth Mlowe

Computing details

Data collection: APEX3 (Bruker, 2017); cell refinement: SAINT (Bruker, 2017); data reduction: SAINT (Bruker, 2017); program(s) used to solve structure: SHELXS (Sheldrick, 2008); program(s) used to refine structure: SHELXL (Sheldrick, 2015); molecular graphics: ORTEP-3 for Windows (Farrugia, 2012); software used to prepare material for publication: PLATON (Spek, 2020).

(3Z)-7-Methoxy-3-(2-phenylhydrazinylidene)-1-benzofuran-2(3H)-one

Crystal data

$\mathrm{C}_{15} \mathrm{H}_{12} \mathrm{~N}_{2} \mathrm{O}_{3}$

$M_{r}=268.27$

Monoclinic, $C 2 / c$

$a=17.436(2) \AA$

$b=7.2485$ (7) $\AA$

$c=20.595(2) \AA$

$\beta=99.181(4)^{\circ}$

$V=2569.6(5) \AA^{3}$

$Z=8$

\section{Data collection}

Bruker APEXII CCD

diffractometer

$\varphi$ and $\omega$ scans

Absorption correction: multi-scan

(SADABS; Krause et al., 2015)

$T_{\min }=0.629, T_{\max }=0.745$

12800 measured reflections

Refinement

Refinement on $F^{2}$

Least-squares matrix: full

$R\left[F^{2}>2 \sigma\left(F^{2}\right)\right]=0.073$

$w R\left(F^{2}\right)=0.152$

$S=1.01$

2427 reflections

187 parameters

0 restraints
$F(000)=1120$

$D_{\mathrm{x}}=1.387 \mathrm{Mg} \mathrm{m}^{-3}$

Mo $K \alpha$ radiation, $\lambda=0.71073 \AA$

Cell parameters from 2240 reflections

$\theta=2.4-26.3^{\circ}$

$\mu=0.10 \mathrm{~mm}^{-1}$

$T=296 \mathrm{~K}$

Prism, colourless

$0.49 \times 0.15 \times 0.06 \mathrm{~mm}$

2427 independent reflections

1463 reflections with $I>2 \sigma(I)$

$R_{\text {int }}=0.085$

$\theta_{\text {max }}=26.0^{\circ}, \theta_{\min }=2.0^{\circ}$

$h=-21 \rightarrow 21$

$k=-8 \rightarrow 8$

$l=-25 \rightarrow 25$

Primary atom site location: difference Fourier map

Secondary atom site location: difference Fourier map

Hydrogen site location: mixed

$\mathrm{H}$ atoms treated by a mixture of independent and constrained refinement

$w=1 /\left[\sigma^{2}\left(F_{\mathrm{o}}^{2}\right)+(0.0517 P)^{2}+2.9831 P\right]$

where $P=\left(F_{\mathrm{o}}{ }^{2}+2 F_{\mathrm{c}}{ }^{2}\right) / 3$ 
$(\Delta / \sigma)_{\max }<0.001$

$\Delta \rho_{\max }=0.18 \mathrm{e} \AA^{-3}$

$\Delta \rho_{\min }=-0.18$ e $\AA^{-3}$
Extinction correction: SHELXL2016/6

(Sheldrick 2015),

$\mathrm{Fc}^{*}=\mathrm{kFc}\left[1+0.001 \mathrm{xFc}^{2} \lambda^{3} / \sin (2 \theta)\right]^{-1 / 4}$

Extinction coefficient: $0.0015(4)$

\section{Special details}

Geometry. All esds (except the esd in the dihedral angle between two 1.s. planes) are estimated using the full covariance matrix. The cell esds are taken into account individually in the estimation of esds in distances, angles and torsion angles; correlations between esds in cell parameters are only used when they are defined by crystal symmetry. An approximate (isotropic) treatment of cell esds is used for estimating esds involving l.s. planes.

Fractional atomic coordinates and isotropic or equivalent isotropic displacement parameters $\left(\AA^{2}\right)$

\begin{tabular}{|c|c|c|c|c|}
\hline & $x$ & $y$ & $z$ & $U_{\text {iso }} * / U_{\text {eq }}$ \\
\hline $\mathrm{O} 1$ & $0.42001(12)$ & $0.2382(3)$ & $0.41831(10)$ & $0.0546(6)$ \\
\hline $\mathrm{O} 2$ & $0.31175(13)$ & 0.3077 (4) & $0.45997(10)$ & $0.0648(7)$ \\
\hline $\mathrm{O} 3$ & $0.53382(14)$ & 0.1359 (4) & $0.34426(11)$ & $0.0728(8)$ \\
\hline N1 & $0.42389(15)$ & $0.3396(4)$ & $0.58811(12)$ & 0.0477 (7) \\
\hline N2 & $0.35184(16)$ & 0.3699 (4) & $0.59750(13)$ & $0.0500(7)$ \\
\hline $\mathrm{C} 1$ & 0.38107 (19) & $0.2841(5)$ & $0.46932(14)$ & $0.0483(8)$ \\
\hline $\mathrm{C} 2$ & $0.43742(17)$ & 0.2955 (4) & 0.52959 (13) & $0.0434(8)$ \\
\hline $\mathrm{C} 3$ & $0.51208(17)$ & $0.2536(4)$ & 0.51198 (14) & $0.0450(8)$ \\
\hline $\mathrm{C} 4$ & $0.58718(18)$ & $0.2419(5)$ & $0.54600(16)$ & $0.0591(10)$ \\
\hline $\mathrm{H} 4 \mathrm{~A}$ & 0.598100 & 0.265759 & 0.590895 & $0.071^{*}$ \\
\hline $\mathrm{C} 5$ & $0.64493(19)$ & $0.1937(5)$ & $0.51096(18)$ & $0.0653(10)$ \\
\hline H5A & 0.695811 & 0.185900 & 0.532762 & $0.078^{*}$ \\
\hline $\mathrm{C} 6$ & $0.6297(2)$ & $0.1564(5)$ & $0.44427(17)$ & $0.0615(10)$ \\
\hline H6A & 0.670362 & 0.122386 & 0.422508 & $0.074 *$ \\
\hline $\mathrm{C} 7$ & $0.55574(19)$ & $0.1688(5)$ & $0.40953(15)$ & $0.0521(9)$ \\
\hline $\mathrm{C} 8$ & $0.49847(17)$ & $0.2183(4)$ & $0.44538(14)$ & $0.0466(8)$ \\
\hline C9 & $0.5936(2)$ & $0.0804(6)$ & $0.30818(17)$ & $0.0778(12)$ \\
\hline H9A & 0.571774 & 0.063916 & 0.262729 & $0.117^{*}$ \\
\hline H9B & 0.615969 & -0.033713 & 0.325700 & $0.117^{*}$ \\
\hline $\mathrm{H} 9 \mathrm{C}$ & 0.633113 & 0.173711 & 0.311913 & $0.117^{*}$ \\
\hline $\mathrm{C} 10$ & $0.33773(18)$ & $0.4270(4)$ & $0.65966(14)$ & $0.0467(8)$ \\
\hline C11 & $0.3957(2)$ & $0.4326(5)$ & $0.71354(14)$ & $0.0594(10)$ \\
\hline H11A & 0.445790 & 0.395598 & 0.709579 & $0.071^{*}$ \\
\hline C12 & $0.3796(2)$ & $0.4926(5)$ & $0.77285(16)$ & $0.0671(11)$ \\
\hline H12A & 0.419310 & 0.497293 & 0.808808 & $0.081^{*}$ \\
\hline C13 & $0.3062(2)$ & $0.5458(5)$ & $0.78029(16)$ & $0.0640(10)$ \\
\hline H13A & 0.295701 & 0.585028 & 0.820967 & $0.077 *$ \\
\hline C14 & $0.2482(2)$ & $0.5402(5)$ & $0.72659(16)$ & $0.0604(10)$ \\
\hline H14A & 0.198194 & 0.577300 & 0.730814 & $0.073^{*}$ \\
\hline C15 & $0.26373(19)$ & $0.4799(5)$ & $0.66640(15)$ & $0.0538(9)$ \\
\hline H15A & 0.224071 & 0.475136 & 0.630425 & $0.065^{*}$ \\
\hline $\mathrm{H} 1$ & $0.313(2)$ & $0.352(5)$ & $0.5627(17)$ & $0.082(13)^{*}$ \\
\hline
\end{tabular}


Atomic displacement parameters $\left(\AA^{2}\right)$

\begin{tabular}{lllllll}
\hline & $U^{11}$ & $U^{22}$ & $U^{33}$ & $U^{12}$ & $U^{13}$ & $U^{23}$ \\
\hline O1 & $0.0484(13)$ & $0.0750(18)$ & $0.0406(11)$ & $-0.0036(12)$ & $0.0072(10)$ & $-0.0031(11)$ \\
O2 & $0.0484(15)$ & $0.092(2)$ & $0.0533(14)$ & $-0.0061(13)$ & $0.0049(11)$ & $-0.0059(12)$ \\
O3 & $0.0659(16)$ & $0.103(2)$ & $0.0527(14)$ & $0.0041(14)$ & $0.0200(12)$ & $-0.0074(14)$ \\
N1 & $0.0488(16)$ & $0.0505(18)$ & $0.0441(14)$ & $-0.0068(13)$ & $0.0082(12)$ & $0.0010(12)$ \\
N2 & $0.0471(17)$ & $0.060(2)$ & $0.0427(15)$ & $-0.0017(14)$ & $0.0062(13)$ & $-0.0044(13)$ \\
C1 & $0.049(2)$ & $0.054(2)$ & $0.0428(17)$ & $-0.0112(17)$ & $0.0118(15)$ & $0.0010(15)$ \\
C2 & $0.0476(18)$ & $0.044(2)$ & $0.0375(16)$ & $-0.0077(15)$ & $0.0050(14)$ & $0.0020(14)$ \\
C3 & $0.0498(19)$ & $0.042(2)$ & $0.0431(16)$ & $-0.0085(15)$ & $0.0082(14)$ & $0.0052(14)$ \\
C4 & $0.053(2)$ & $0.073(3)$ & $0.0489(18)$ & $-0.0091(18)$ & $0.0014(16)$ & $0.0038(17)$ \\
C5 & $0.044(2)$ & $0.083(3)$ & $0.068(2)$ & $-0.0021(18)$ & $0.0070(18)$ & $0.012(2)$ \\
C6 & $0.054(2)$ & $0.067(3)$ & $0.067(2)$ & $-0.0014(18)$ & $0.0194(18)$ & $0.0100(19)$ \\
C7 & $0.058(2)$ & $0.051(2)$ & $0.0499(19)$ & $-0.0049(17)$ & $0.0159(17)$ & $0.0025(16)$ \\
C8 & $0.0481(19)$ & $0.047(2)$ & $0.0447(17)$ & $-0.0073(15)$ & $0.0070(15)$ & $0.0063(15)$ \\
C9 & $0.092(3)$ & $0.084(3)$ & $0.066(2)$ & $0.011(2)$ & $0.038(2)$ & $-0.006(2)$ \\
C10 & $0.052(2)$ & $0.047(2)$ & $0.0404(16)$ & $-0.0068(15)$ & $0.0080(15)$ & $-0.0010(15)$ \\
C11 & $0.055(2)$ & $0.076(3)$ & $0.0456(18)$ & $0.0031(18)$ & $0.0016(16)$ & $-0.0101(17)$ \\
C12 & $0.074(3)$ & $0.080(3)$ & $0.0446(19)$ & $0.003(2)$ & $0.0019(18)$ & $-0.0106(18)$ \\
C13 & $0.078(3)$ & $0.069(3)$ & $0.047(2)$ & $-0.002(2)$ & $0.0174(19)$ & $-0.0081(17)$ \\
C14 & $0.060(2)$ & $0.061(3)$ & $0.065(2)$ & $0.0041(18)$ & $0.0248(19)$ & $0.0021(18)$ \\
C15 & $0.050(2)$ & $0.060(2)$ & $0.0511(19)$ & $-0.0028(17)$ & $0.0061(16)$ & $0.0037(16)$ \\
& & & & & & \\
\hline
\end{tabular}

Geometric parameters $\left(\AA,{ }^{\circ}\right)$

\begin{tabular}{llll}
\hline $\mathrm{O} 1-\mathrm{C} 1$ & $1.380(3)$ & $\mathrm{C} 6-\mathrm{C} 7$ & $1.375(5)$ \\
$\mathrm{O} 1-\mathrm{C} 8$ & $1.400(3)$ & $\mathrm{C} 6-\mathrm{H} 6 \mathrm{~A}$ & 0.9300 \\
$\mathrm{O} 2-\mathrm{C} 1$ & $1.205(3)$ & $\mathrm{C} 7-\mathrm{C} 8$ & $1.381(4)$ \\
$\mathrm{O} 3-\mathrm{C} 7$ & $1.359(4)$ & $\mathrm{C} 9-\mathrm{H} 9 \mathrm{~A}$ & 0.9600 \\
$\mathrm{O} 3-\mathrm{C} 9$ & $1.431(4)$ & $\mathrm{C} 9-\mathrm{H} 9 \mathrm{~B}$ & 0.9600 \\
$\mathrm{~N} 1-\mathrm{C} 2$ & $1.304(3)$ & $\mathrm{C} 9-\mathrm{H} 9 \mathrm{C}$ & 0.9600 \\
$\mathrm{~N} 1-\mathrm{N} 2$ & $1.320(3)$ & $\mathrm{C} 10-\mathrm{C} 15$ & $1.374(4)$ \\
$\mathrm{N} 2-\mathrm{C} 10$ & $1.404(4)$ & $\mathrm{C} 10-\mathrm{C} 11$ & $1.377(4)$ \\
$\mathrm{N} 2-\mathrm{H} 1$ & $0.92(4)$ & $\mathrm{C} 11-\mathrm{C} 12$ & $1.367(4)$ \\
$\mathrm{C} 1-\mathrm{C} 2$ & $1.457(4)$ & $\mathrm{C} 11-\mathrm{H} 11 \mathrm{~A}$ & 0.9300 \\
$\mathrm{C} 2-\mathrm{C} 3$ & $1.438(4)$ & $\mathrm{C} 12-\mathrm{C} 13$ & $1.369(5)$ \\
$\mathrm{C} 3-\mathrm{C} 8$ & $1.378(4)$ & $\mathrm{C} 12-\mathrm{H} 12 \mathrm{~A}$ & 0.9300 \\
$\mathrm{C} 3-\mathrm{C} 4$ & $1.386(4)$ & $\mathrm{C} 13-\mathrm{C} 14$ & $1.375(5)$ \\
$\mathrm{C} 4-\mathrm{C} 5$ & $1.374(4)$ & $\mathrm{C} 13-\mathrm{H} 13 \mathrm{~A}$ & 0.9300 \\
$\mathrm{C} 4-\mathrm{H} 4 \mathrm{~A}$ & 0.9300 & $\mathrm{C} 14-\mathrm{C} 15$ & $1.381(4)$ \\
$\mathrm{C} 5-\mathrm{C} 6$ & $1.383(5)$ & $\mathrm{C} 14-\mathrm{H} 14 \mathrm{~A}$ & 0.9300 \\
$\mathrm{C} 5-\mathrm{H} 5 \mathrm{~A}$ & 0.9300 & $\mathrm{C} 15-\mathrm{H} 15 \mathrm{~A}$ & 0.9300 \\
& & & \\
$\mathrm{C} 1-\mathrm{O} 1-\mathrm{C} 8$ & $106.9(2)$ & $\mathrm{C} 3-\mathrm{C} 8-\mathrm{C} 7$ & $123.8(3)$ \\
$\mathrm{C} 7-\mathrm{O} 3-\mathrm{C} 9$ & $116.7(3)$ & $\mathrm{C} 3-\mathrm{C} 8-\mathrm{O} 1$ & $112.3(3)$ \\
$\mathrm{C} 2-\mathrm{N} 1-\mathrm{N} 2$ & $119.5(3)$ & $\mathrm{C} 7-\mathrm{C} 8-\mathrm{O} 1$ & $123.9(3)$ \\
$\mathrm{N} 1-\mathrm{N} 2-\mathrm{C} 10$ & $119.5(3)$ & $\mathrm{O} 3-\mathrm{C} 9-\mathrm{H} 9 \mathrm{~A}$ & 109.5
\end{tabular}




\begin{tabular}{|c|c|c|c|}
\hline $\mathrm{N} 1-\mathrm{N} 2-\mathrm{H} 1$ & $118(2)$ & $\mathrm{O} 3-\mathrm{C} 9-\mathrm{H} 9 \mathrm{~B}$ & 109.5 \\
\hline $\mathrm{C} 10-\mathrm{N} 2-\mathrm{H} 1$ & $123(2)$ & $\mathrm{H} 9 \mathrm{~A}-\mathrm{C} 9-\mathrm{H} 9 \mathrm{~B}$ & 109.5 \\
\hline $\mathrm{O} 2-\mathrm{C} 1-\mathrm{O} 1$ & $121.0(3)$ & $\mathrm{O} 3-\mathrm{C} 9-\mathrm{H} 9 \mathrm{C}$ & 109.5 \\
\hline $\mathrm{O} 2-\mathrm{C} 1-\mathrm{C} 2$ & $130.6(3)$ & $\mathrm{H} 9 \mathrm{~A}-\mathrm{C} 9-\mathrm{H} 9 \mathrm{C}$ & 109.5 \\
\hline $\mathrm{O} 1-\mathrm{C} 1-\mathrm{C} 2$ & $108.4(3)$ & $\mathrm{H} 9 \mathrm{~B}-\mathrm{C} 9-\mathrm{H} 9 \mathrm{C}$ & 109.5 \\
\hline $\mathrm{N} 1-\mathrm{C} 2-\mathrm{C} 3$ & $126.1(3)$ & $\mathrm{C} 15-\mathrm{C} 10-\mathrm{C} 11$ & $119.4(3)$ \\
\hline $\mathrm{N} 1-\mathrm{C} 2-\mathrm{C} 1$ & $127.2(3)$ & $\mathrm{C} 15-\mathrm{C} 10-\mathrm{N} 2$ & $118.6(3)$ \\
\hline $\mathrm{C} 3-\mathrm{C} 2-\mathrm{C} 1$ & $106.7(2)$ & $\mathrm{C} 11-\mathrm{C} 10-\mathrm{N} 2$ & $122.1(3)$ \\
\hline $\mathrm{C} 8-\mathrm{C} 3-\mathrm{C} 4$ & $119.4(3)$ & $\mathrm{C} 12-\mathrm{C} 11-\mathrm{C} 10$ & $120.1(3)$ \\
\hline $\mathrm{C} 8-\mathrm{C} 3-\mathrm{C} 2$ & $105.7(3)$ & $\mathrm{C} 12-\mathrm{C} 11-\mathrm{H} 11 \mathrm{~A}$ & 120.0 \\
\hline $\mathrm{C} 4-\mathrm{C} 3-\mathrm{C} 2$ & $134.9(3)$ & $\mathrm{C} 10-\mathrm{C} 11-\mathrm{H} 11 \mathrm{~A}$ & 120.0 \\
\hline $\mathrm{C} 5-\mathrm{C} 4-\mathrm{C} 3$ & $117.6(3)$ & $\mathrm{C} 11-\mathrm{C} 12-\mathrm{C} 13$ & $121.2(3)$ \\
\hline $\mathrm{C} 5-\mathrm{C} 4-\mathrm{H} 4 \mathrm{~A}$ & 121.2 & $\mathrm{C} 11-\mathrm{C} 12-\mathrm{H} 12 \mathrm{~A}$ & 119.4 \\
\hline $\mathrm{C} 3-\mathrm{C} 4-\mathrm{H} 4 \mathrm{~A}$ & 121.2 & $\mathrm{C} 13-\mathrm{C} 12-\mathrm{H} 12 \mathrm{~A}$ & 119.4 \\
\hline $\mathrm{C} 4-\mathrm{C} 5-\mathrm{C} 6$ & $122.1(3)$ & $\mathrm{C} 12-\mathrm{C} 13-\mathrm{C} 14$ & $118.8(3)$ \\
\hline $\mathrm{C} 4-\mathrm{C} 5-\mathrm{H} 5 \mathrm{~A}$ & 119.0 & $\mathrm{C} 12-\mathrm{C} 13-\mathrm{H} 13 \mathrm{~A}$ & 120.6 \\
\hline $\mathrm{C} 6-\mathrm{C} 5-\mathrm{H} 5 \mathrm{~A}$ & 119.0 & $\mathrm{C} 14-\mathrm{C} 13-\mathrm{H} 13 \mathrm{~A}$ & 120.6 \\
\hline $\mathrm{C} 7-\mathrm{C} 6-\mathrm{C} 5$ & $121.3(3)$ & $\mathrm{C} 13-\mathrm{C} 14-\mathrm{C} 15$ & $120.5(3)$ \\
\hline $\mathrm{C} 7-\mathrm{C} 6-\mathrm{H} 6 \mathrm{~A}$ & 119.4 & $\mathrm{C} 13-\mathrm{C} 14-\mathrm{H} 14 \mathrm{~A}$ & 119.8 \\
\hline $\mathrm{C} 5-\mathrm{C} 6-\mathrm{H} 6 \mathrm{~A}$ & 119.4 & $\mathrm{C} 15-\mathrm{C} 14-\mathrm{H} 14 \mathrm{~A}$ & 119.8 \\
\hline $\mathrm{O} 3-\mathrm{C} 7-\mathrm{C} 6$ & $126.6(3)$ & $\mathrm{C} 10-\mathrm{C} 15-\mathrm{C} 14$ & $120.0(3)$ \\
\hline $\mathrm{O} 3-\mathrm{C} 7-\mathrm{C} 8$ & $117.5(3)$ & $\mathrm{C} 10-\mathrm{C} 15-\mathrm{H} 15 \mathrm{~A}$ & 120.0 \\
\hline $\mathrm{C} 6-\mathrm{C} 7-\mathrm{C} 8$ & $115.9(3)$ & $\mathrm{C} 14-\mathrm{C} 15-\mathrm{H} 15 \mathrm{~A}$ & 120.0 \\
\hline $\mathrm{C} 2-\mathrm{N} 1-\mathrm{N} 2-\mathrm{C} 10$ & $-176.6(3)$ & $\mathrm{C} 4-\mathrm{C} 3-\mathrm{C} 8-\mathrm{C} 7$ & $0.8(5)$ \\
\hline $\mathrm{C} 8-\mathrm{O} 1-\mathrm{C} 1-\mathrm{O} 2$ & $-179.2(3)$ & $\mathrm{C} 2-\mathrm{C} 3-\mathrm{C} 8-\mathrm{C} 7$ & $-178.7(3)$ \\
\hline $\mathrm{C} 8-\mathrm{O} 1-\mathrm{C} 1-\mathrm{C} 2$ & $0.8(3)$ & $\mathrm{C} 4-\mathrm{C} 3-\mathrm{C} 8-\mathrm{O} 1$ & $-179.3(3)$ \\
\hline $\mathrm{N} 2-\mathrm{N} 1-\mathrm{C} 2-\mathrm{C} 3$ & $-178.2(3)$ & $\mathrm{C} 2-\mathrm{C} 3-\mathrm{C} 8-\mathrm{O} 1$ & $1.2(4)$ \\
\hline $\mathrm{N} 2-\mathrm{N} 1-\mathrm{C} 2-\mathrm{C} 1$ & $3.7(5)$ & $\mathrm{O} 3-\mathrm{C} 7-\mathrm{C} 8-\mathrm{C} 3$ & $179.2(3)$ \\
\hline $\mathrm{O} 2-\mathrm{C} 1-\mathrm{C} 2-\mathrm{N} 1$ & $-1.8(6)$ & $\mathrm{C} 6-\mathrm{C} 7-\mathrm{C} 8-\mathrm{C} 3$ & $-0.3(5)$ \\
\hline $\mathrm{O} 1-\mathrm{C} 1-\mathrm{C} 2-\mathrm{N} 1$ & $178.3(3)$ & $\mathrm{O} 3-\mathrm{C} 7-\mathrm{C} 8-\mathrm{O} 1$ & $-0.6(5)$ \\
\hline $\mathrm{O} 2-\mathrm{C} 1-\mathrm{C} 2-\mathrm{C} 3$ & $179.9(4)$ & $\mathrm{C} 6-\mathrm{C} 7-\mathrm{C} 8-\mathrm{O} 1$ & $179.8(3)$ \\
\hline $\mathrm{O} 1-\mathrm{C} 1-\mathrm{C} 2-\mathrm{C} 3$ & $-0.1(3)$ & $\mathrm{C} 1-\mathrm{O} 1-\mathrm{C} 8-\mathrm{C} 3$ & $-1.3(3)$ \\
\hline $\mathrm{N} 1-\mathrm{C} 2-\mathrm{C} 3-\mathrm{C} 8$ & $-179.0(3)$ & $\mathrm{C} 1-\mathrm{O} 1-\mathrm{C} 8-\mathrm{C} 7$ & $178.6(3)$ \\
\hline $\mathrm{C} 1-\mathrm{C} 2-\mathrm{C} 3-\mathrm{C} 8$ & $-0.7(3)$ & $\mathrm{N} 1-\mathrm{N} 2-\mathrm{C} 10-\mathrm{C} 15$ & $172.0(3)$ \\
\hline $\mathrm{N} 1-\mathrm{C} 2-\mathrm{C} 3-\mathrm{C} 4$ & $1.5(6)$ & $\mathrm{N} 1-\mathrm{N} 2-\mathrm{C} 10-\mathrm{C} 11$ & $-7.6(5)$ \\
\hline $\mathrm{C} 1-\mathrm{C} 2-\mathrm{C} 3-\mathrm{C} 4$ & $179.9(4)$ & $\mathrm{C} 15-\mathrm{C} 10-\mathrm{C} 11-\mathrm{C} 12$ & $-0.8(5)$ \\
\hline $\mathrm{C} 8-\mathrm{C} 3-\mathrm{C} 4-\mathrm{C} 5$ & $-0.4(5)$ & $\mathrm{N} 2-\mathrm{C} 10-\mathrm{C} 11-\mathrm{C} 12$ & $178.7(3)$ \\
\hline $\mathrm{C} 2-\mathrm{C} 3-\mathrm{C} 4-\mathrm{C} 5$ & $178.9(4)$ & $\mathrm{C} 10-\mathrm{C} 11-\mathrm{C} 12-\mathrm{C} 13$ & $0.8(6)$ \\
\hline $\mathrm{C} 3-\mathrm{C} 4-\mathrm{C} 5-\mathrm{C} 6$ & $-0.5(6)$ & $\mathrm{C} 11-\mathrm{C} 12-\mathrm{C} 13-\mathrm{C} 14$ & $-0.7(6)$ \\
\hline $\mathrm{C} 4-\mathrm{C} 5-\mathrm{C} 6-\mathrm{C} 7$ & $1.0(6)$ & $\mathrm{C} 12-\mathrm{C} 13-\mathrm{C} 14-\mathrm{C} 15$ & $0.7(5)$ \\
\hline $\mathrm{C} 9-\mathrm{O} 3-\mathrm{C} 7-\mathrm{C} 6$ & $0.9(5)$ & $\mathrm{C} 11-\mathrm{C} 10-\mathrm{C} 15-\mathrm{C} 14$ & $0.8(5)$ \\
\hline $\mathrm{C} 9-\mathrm{O} 3-\mathrm{C} 7-\mathrm{C} 8$ & $-178.5(3)$ & $\mathrm{N} 2-\mathrm{C} 10-\mathrm{C} 15-\mathrm{C} 14$ & $-178.8(3)$ \\
\hline $\mathrm{C} 5-\mathrm{C} 6-\mathrm{C} 7-\mathrm{O} 3$ & $179.9(3)$ & $\mathrm{C} 13-\mathrm{C} 14-\mathrm{C} 15-\mathrm{C} 10$ & $-0.8(5)$ \\
\hline $\mathrm{C} 5-\mathrm{C} 6-\mathrm{C} 7-\mathrm{C} 8$ & $-0.6(5)$ & & \\
\hline
\end{tabular}


Hydrogen-bond geometry $\left(\AA,{ }^{\circ}\right)$

$\mathrm{Cg} 3$ is the centroid of the $\mathrm{C} 10-\mathrm{C} 15$ phenyl ring.

\begin{tabular}{lllll}
\hline$D-\mathrm{H} \cdots A$ & $D-\mathrm{H}$ & $\mathrm{H} \cdots A$ & $D \cdots A$ & $D-\mathrm{H} \cdots A$ \\
\hline $\mathrm{N} 2-\mathrm{H} 1 \cdots \mathrm{O} 2$ & $0.92(4)$ & $2.14(3)$ & $2.843(3)$ & $133(3)$ \\
$\mathrm{N} 2-\mathrm{H} 1 \cdots \mathrm{O} 2^{\mathrm{i}}$ & $0.92(4)$ & $2.44(4)$ & $3.181(4)$ & $138(3)$ \\
$\mathrm{C} 9-\mathrm{H} 9 C \cdots C g 3^{\mathrm{ii}}$ & 0.96 & 2.70 & $3.555(4)$ & 149 \\
\hline
\end{tabular}

Symmetry codes: (i) $-x+1 / 2,-y+1 / 2,-z+1$; (ii) $-x+1,-y+1,-z+1$.

Percentage contributions of interatomic contacts to the Hirshfeld surface for the title compound

\begin{tabular}{ll}
\hline Contact & Percentage contribution \\
$\mathrm{H} \cdots \mathrm{H}$ & 40.7 \\
$\mathrm{O} \cdots \mathrm{H} / \mathrm{H} \cdots \mathrm{O}$ & 24.7 \\
$\mathrm{C} \cdots \mathrm{H} / \mathrm{H} \cdots \mathrm{C}$ & 16.1 \\
$\mathrm{C} \cdots \mathrm{C}$ & 8.8 \\
$\mathrm{~N} \cdots \mathrm{C} / \mathrm{C} \cdots \mathrm{N}$ & 3.8 \\
$\mathrm{~N} \cdots \mathrm{H} / \mathrm{H} \cdots \mathrm{N}$ & 3.5 \\
$\mathrm{O} \cdots \mathrm{C} / \mathrm{C} \cdots \mathrm{O}$ & 1.9 \\
$\mathrm{O} \cdots \mathrm{N} / \mathrm{N} \cdots \mathrm{O}$ & 0.4 \\
$\mathrm{O} \cdots \mathrm{O}$ & 0.2
\end{tabular}

